\title{
The Pluralistic View of Politics: Asymmetric Lobbyists, Ideological Uncertainty and Political Entry
}

\author{
David Martimort ${ }^{a}$, Aggey Semenov ${ }^{b}$ \\ ${ }^{a}$ University of Toulouse and Institut Universitaire de France, Manufacture des Tabacs, \\ 21 Allee de Brienne, 31000 Toulouse, France. \\ ${ }^{b}$ Corresponding author: National University of Singapore, Department of Economics, \\ Faculty of Arts and Social Sciences, AS2 Level 6, 1 Arts Link, 117570 Singapore. Tel. \\ $(++65) 6516$ 6010. Fax (65) 6775 2646. E-mail: ecssa@nus.edu.sg
}

This version: 19 th October 2006

\begin{abstract}
In a spatial common agency model, two asymmetric interest groups (principals) influence a unique decision-maker (agent). The decision-maker chooses a onedimensional policy on behalf of those principals and has private information about his ideology after contracting with principals. The policy is always efficient and reflects a weighted average between the principals' and the agent's ideal points. The decisionmaker's expected rent can be fully extracted by congruent interest groups but not when interest groups' objectives are too conflicting. The degree of congruence is endogenous. It depends on the level of ideological uncertainty, whether interest groups can easily buy influence or not, and on their relative ideological distance with the principal. In particular, the game of influence is biased towards the closest principal. This suggests that free-entry equilibria of a lobbying game may only reflect the full diversity of the lobbying groups' preferences when there is enough ideological uncertainty and when those groups are close to be equally distant to the decision-maker.
\end{abstract}

Keywords: Lobbying, Common Agency, Ideological Uncertainty, Pluralistic Politics.

JEL Classification: D72; D82. 


\section{Introduction}

Following Grossman and Helpman (1994), ${ }^{1}$ it is by now common to rely on the so-called common agency model of politics to analyze the influence of interest groups in the political arena. In that paradigm, competing lobbying groups (the principals) design noncooperatively policy contingent contributions to influence a common decision-maker (the agent). The decision-maker chooses which contributions to accept and then which policy to implement in response to those contributions.

Under complete information, this decentralized political process reaches efficiency, i.e., the aggregate payoff of the grand-coalition made of all principals and their common agent is maximized. Efficiency comes of course at no surprise. Some version of the Coase Theorem applies and the overall bargaining between interest groups and the decision-maker should be efficient. In fact, the game of influence between interest groups only matters in so far that it might generate different distributions of the political surplus between the different interest groups and the decision-maker. Although, they all implement the same efficient allocation once enough concavity is assumed, there may exist a whole multiplicity of equilibria of the common agency game which differs in terms of these distributions. In complete information environments, the equilibrium distributions of the surplus are readily characterized by means of simple inequalities (Bernheim and Whinston, 1986; Laussel and Le Breton, 2001). Such characterization matters for two reasons. First, it helps to find reasons why political principals may pit one group against the others and withdraw some rent from the political process. Second, this characterization delineates also conditions under which entering the political process is a valuable strategy for interests groups, in particular when they face a fixed-cost of entry as argued by Mitra (1999). This payoff characterization provides thus a way to endogenize the active groups in the polity. This is an important insight to confirm or not the pluralistic view of politics which has been pushed forward by a whole branch of the political science literature mainly associated with Dahl (1961).

However, little is known about payoff characterization in incomplete information environments. ${ }^{2}$ The characterization of the interest groups' payoffs under ideological uncertainty is an important step of the analysis of entry in the political arena. Indeed, interest groups exert influence in an environment plagued by much economic uncertainty on the exact policy that political decision-makers would like to follow. ${ }^{3}$

Following most of the political science literature, ${ }^{4}$ we are interested in describing the

\footnotetext{
${ }^{1}$ See also Bernheim and Whinston (1986).

${ }^{2}$ Laussel and Le Breton (1998) provides such characterization in the context of public good provision.

${ }^{3}$ Horn (1995).

${ }^{4}$ See Austen-Smith and Banks (2000) among others.
} 
equilibrium behavior of two interest groups and a decision-maker all having spatial preferences with ideal points in a one-dimensional policy space. To describe competition for the agent's services, competing interest groups have ideal points located asymmetrically on each side of the agent's ideal point. To model some form of private information, the agent learns his own ideal point in the policy space after contracting with principals. This is meant to capture the uncertainty on the ideological bias of key decision-makers in the political process. Principals non-cooperatively design contributions not only to influence the agent's choice as it would be the case under complete information, but also may want to do so to elicit the decision-maker's ideal points.

Under ex ante contracting, principals can commit themselves to contributions before the agent learns his ideal point. Contracting takes thus place under symmetric but incomplete information. Indeed, interest groups exert pressures at various stages of the policy process. Key policy-makers (like Congressmen or civil servants) are often subject to the influence of lobbying groups at the policy inception stage, i.e., ex ante before they learn their most preferred policy. ${ }^{5}$ The equilibrium policy which results from these pressures reflects the relative political power of interest groups and how effective is their influence on the political process.

Because of symmetric information at the contracting stage, the equilibrium outcome remains always efficient, whatever the realization of the agent's ideal point. Equilibrium contributions are truthful, i.e., reflect the principals' marginal preferences on policy. ${ }^{6}$ With truthful contributions, principals compete to attract the agent's services by playing on the fixed-fees of those schedules since marginal contributions are fully determined by the principals' preferences only. Whether there is fierce conflict or more congruence between principals depends on the parameters of the model:

- The extent of ideological uncertainty. When there is little ideological uncertainty compared to the degree of polarization, interest groups compete head-to-head for the decisionmaker's services. The latter enjoys some positive ex ante rent out of the political process by playing one principal against the other. Instead, when ideological uncertainty is more severe, interest groups jointly extract the decision-maker's expected rent and appropriate the whole surplus of the political process.

- The ideological bias of the decision-maker. When the decision-maker is more ideologically motivated, contributions are less useful and the decision-maker does not get any rent. On the contrary if the agent is easier to bribe he might appropriate part of the political surplus.

- The degree of asymmetry between the principals. The more asymmetric are the principals

\footnotetext{
${ }^{5}$ See Martimort and Semenov (2006) for the case of ex-post contracting.

${ }^{6} \mathrm{~A}$ similar result has also been obtained in a public good context by Laussel and Le Breton (1998).
} 
the easier it is for the decision-maker to pit one against the other. The more ideologically distant interest groups get hurt by this competition and, as a result, might prefer to stay out of the political process in model where entry is endogenous.

\section{The Model}

Two principals $P_{1}$ and $P_{2}$ simultaneously offer contributions to influence a decision-maker (or common agent). Those principals can be thought of as two legislative committees willing to influence a common bureaucrat (a regulatory agency for instance) or as two lobbying groups dealing with an elected political decision-maker. We denote by $q \in \mathbb{R}$ a one-dimensional policy parameter over which the decision-maker has control. This can be a regulated price, an import tariff or an export subsidy, a wage level or a number of permits depending on the application that one has in mind. Principal $P_{i}(i=1,2)$ has a quasi-linear utility function over policies and monetary transfers $t_{i}$ which is given by:

$$
V_{i}\left(q, t_{i}\right)=-\frac{1}{2}\left(q-a_{i}\right)^{2}-t_{i}
$$

The parameter $a_{i}$ is $P_{i}$ 's ideal point in the one-dimensional policy space. We consider an asymmetric environment so that principals' ideal points are asymmetrically located around the origin, $a_{1}=a+h$ and $a_{2}=-a .^{7}$ These parameters $a$ and $h$ can be viewed respectively as the degree of polarization and a measure of the decision-maker's bias towards principal 2. The common agent has similar quasi-linear preferences:

$$
U\left(q, \sum_{i=1}^{2} t_{i}, \theta\right)=-\frac{\beta}{2}(q-\theta)^{2}+\sum_{i=1}^{2} t_{i} .
$$

The agent's ideal point $\theta$ is uniformly distributed on an interval $\Theta=[-\delta, \delta]$, centered around zero. $^{8} \delta$ measures the degree of the ideological uncertainty. The non-negative parameter $\beta$ characterizes how the agent trades off contributions against his own ideological bias. As $\beta$ increases, the agent values less monetary transfers and puts more emphasis on ideology.

Interest groups influence the decision-maker by offering nonlinear contributions $t_{i}(q)$ which specify a monetary transfer to the agent depending on the decision $q$ he takes. These contributions might serve not only to influence the decision-maker but also to elicit his ideology.

Timing: We consider a game of delegated common agency where the agent may choose

\footnotetext{
${ }^{7}$ We assume $a, h \geq 0$.

${ }^{8}$ In much of the literature following Grossman and Helpman (1994), this ideal point is viewed as the welfare-maximizing policy and our model is consistent with this interpretation as well.
} 
to accept whatever set of contributions maximizes his payoff. Under ex ante contracting the common agency game unfolds as follows.

- Interest groups non-cooperatively make their offers $t_{i}(q)$ to the agent.

- The agent decides whether to accept or refuse each of these offers. If he refuses all offers, he gets his status quo payoff normalized at zero. ${ }^{9}$

- The agent learns his preferences parameter $\theta$.

- Finally, the agent chooses the policy $q$ and receives the corresponding payments from the principals whose offers have been accepted.

Benchmark: The optimal first-best policy $q^{F B}(\theta)$ which maximizes the grand-coalition payoff satisfies:

$$
q^{F B}(\theta)=\arg \max _{q \in \mathbb{R}}\left\{\sum_{i=1}^{2} V_{i}\left(q, t_{i}\right)+U\left(q, \sum_{i=1}^{2} t_{i}, \theta\right)\right\}=\frac{\beta \theta+h}{\beta+2} .
$$

As the decision-maker's ideology matters more (i.e., $\beta$ increases), the optimal policy is shifted towards the agent's ideal point. Political principals find it more difficult to influence the agent as his ideological bias increases. Nevertheless, this policy always reflects also the existing groups' preferences. As $h>0$, the efficient policy is biased towards $P_{1}$ 's ideal point.

\section{Truthful Equilibria}

Let us denote by $U(\theta)$ the agent's payoff when he accepts both principals' contributions $\left\{t_{1}(\cdot), t_{2}(\cdot)\right\}$ and $q(\theta)$ the corresponding optimal policy chosen by the agent, namely:

$$
U(\theta)=\max _{q \in \mathbb{R}}\left\{\sum_{i=1}^{2} t_{i}(q)-\frac{\beta}{2}(q-\theta)^{2}\right\} \text { and } q(\theta)=\arg \max _{q \in \mathbb{R}}\left\{\sum_{i=1}^{2} t_{i}(q)-\frac{\beta}{2}(q-\theta)^{2}\right\} .
$$

Similarly, the rent-output profile $\left\{U_{i}(\theta), q_{i}(\theta)\right\}$ implemented had the agent only accepted principal $P_{i}$ 's contribution is defined as:

$$
U_{i}(\theta)=\max _{q \in \mathbb{R}}\left\{t_{i}(q)-\frac{\beta}{2}(q-\theta)^{2}\right\} \text { and } q_{i}(\theta)=\arg \max _{q \in \mathbb{R}}\left\{t_{i}(q)-\frac{\beta}{2}(q-\theta)^{2}\right\} .
$$

The following Lemma characterizes the implementable profile $\{U(\theta), q(\theta)\}$.

Lemma 1 : $U(\theta)$ and $q(\theta)$ are a.e. differentiable with, at any differentiability point,

$$
\dot{U}(\theta)=\beta(q(\theta)-\theta), \quad \dot{q}(\theta) \geq 0 .
$$

\footnotetext{
${ }^{9}$ It is important to stress that the agent's outside opportunity if he refuses all contributions is in fact his payoff if he chooses his own ideal policy.
} 
Principal $P_{i}$ 's best-response to any contribution $t_{-i}^{*}(\cdot)$ offered by $P_{-i}$ and accepted by the agent must generate a rent-output profile which solves the following problem:

$$
\begin{gathered}
\left(\mathcal{P}_{i}\right): \max _{\{q(\cdot), U(\cdot)\}} \int_{-\delta}^{\delta}\left\{-\frac{1}{2}\left(q(\theta)-a_{i}\right)^{2}-\frac{\beta}{2}(q(\theta)-\theta)^{2}+t_{-i}^{*}(q(\theta))-U(\theta)\right\} \frac{d \theta}{2 \delta} \\
\text { subject to }(2), \text { and } \\
\int_{-\delta}^{\delta} U(\theta) \frac{d \theta}{2 \delta} \geq \max \left\{0, \int_{-\delta}^{\delta} U_{-i}(\theta) \frac{d \theta}{2 \delta}\right\} .
\end{gathered}
$$

The participation constraint (3) stipulates that the agent prefers to take both contracts rather than either accepting only $P_{-i}$ 's offer or refusing both contributions. Because contracting takes place ex ante, this participation constraint is written in terms of the agent's ex ante rent $\int_{-\delta}^{\delta} U(\theta) \frac{d \theta}{2 \delta}$.

Under ex ante contracting, principal $P_{i}$ 's best-response to any contract $t_{-i}^{*}(\cdot)$ is simply to make the decision-maker residual claimant for the aggregate payoff of the bilateral coalition they form so that the latter chooses the right policy whatever the realization of his ideal point. This is done by offering the following truthful contribution

$$
t_{i}(q)=-\frac{1}{2}\left(q-a_{i}\right)^{2}-C_{i}, \quad \text { for some } C_{i} \in \mathbb{R} .
$$

The constant $C_{i}$ is in fact principal $P_{i}$ 's payoff for any realization of $\theta$. Using this remark, we may look for equilibria in truthful schedules. ${ }^{10}$

To characterize these equilibrium payoffs, we now define the aggregate surplus of a coalition made of principals belonging to any set $S \in\{1,2,\{1,2\}\}$ and the common agent when his ideal point is $\theta$ as:

$$
W_{S}(\theta)=\max _{q \in \mathbb{R}}-\frac{1}{2}\left\{\sum_{i \in S}\left(q-a_{i}\right)^{2}+\beta(q-\theta)^{2}\right\} .
$$

Then let $W_{S}$ be the expected total surplus of such a coalition: $W_{S}=\int_{-\delta}^{\delta} W_{S}(\theta) \frac{d \theta}{2 \delta}$. The properties of the cooperative game with characteristic function $W_{S}$ are important to understand the equilibrium distributions of payoffs between the principals and their agent under ex ante contracting. ${ }^{11}$ Congruent principals are able to jointly extract the ex

\footnotetext{
${ }^{9}$ See Laffont and Martimort (2002, Chapter 2) for instance.

${ }^{10}$ Bernheim and Whinston (1986) suggested to focus on the so-called truthful contributions in delegated common agency games of complete information which are of the form $t_{i}(q)=\max \left\{0,-\frac{1}{2}\left(q-a_{i}\right)^{2}-\right.$ $\left.C_{i}\right\}$, for some $C_{i} \in \mathbb{R}$. The notion of truthful contribution is still relevant under ex ante contracting but must be slightly amended to relax the non-negativity assumption. Although innocuous under complete information, this assumption would actually preclude efficiency under ex ante contracting. In fact, under ex ante contracting, the optimal contribution solving $\left(\mathcal{P}_{i}\right)$ will make the agent residual claimant for maximizing principal $P_{i}^{\prime}$ 's objective. Satisfying altogether the incentive constraint (2) and the participation constraint (3) may require having the agent pay the principal for some realizations of $\theta$ (e.g. when their ideal points are far apart).

${ }^{11}$ This point was already stressed by Laussel and Le Breton (2001).
} 
agent's ante rent whereas principals with conflicting preferences will not. In our model, the congruence between principals is however endogenous and depends on the parameters of the model.

\section{Results and Implications}

We now summarize the main features of truthful equilibria under ex ante contracting.

Proposition 1 : For any degree of ideological uncertainty $\delta$, the decision $q^{e a}(\theta)$ taken by the agent is always efficient from the grand-coalition's viewpoint, $q^{e a}(\theta)=q^{F B}(\theta), \forall \theta \in$ $\Theta$.

Congruent Principals: Assume that $\delta \geq \delta^{*}(a, h, \beta)=\sqrt{\frac{3(\beta+2)}{\beta}(a+h) a+\frac{3 h^{2}}{2 \beta}}$. The agent gets no ex ante rent at any truthful equilibrium. The set of truthful equilibrium payoffs $\left(C_{1}, C_{2}\right)$ for the principals is an interval defined by the following linear constraints:

$$
\begin{gathered}
C_{1}+C_{2}=W_{12}, W_{i} \leq C_{i} \\
W_{i}=-\frac{\beta\left(3 a_{i}^{2}+\delta^{2}\right)}{6(\beta+1)}, \text { and } W_{12}=-a(a+h)-\frac{(\beta+1) h^{2}}{2(\beta+2)}-\frac{\beta \delta^{2}}{3(\beta+2)} .
\end{gathered}
$$

Conflicting Principals: Assume that $\delta<\delta^{*}(a, h, \beta)$. The agent gets a positive rent in the unique truthful equilibrium:

$$
\int_{-\delta}^{\delta} U(\theta) \frac{d \theta}{2 \delta}=\sum_{i=1}^{2} W_{i}-W_{12}>0
$$

The principals $P_{1}$ and $P_{2}$ get the following expected payoffs in this equilibrium

$C_{1}=W_{12}-W_{2}=C_{2}-\frac{\beta h(2 a+h)}{2(\beta+1)} \leq C_{2}$, and $C_{2}=W_{12}-W_{1}=-\frac{3(a(\beta+2)+h)^{2}+\beta^{2} \delta^{2}}{6(\beta+1)(\beta+2)}$.

The first important result of Proposition 1 is that the equilibrium policy is always efficient from the grand-coalition's viewpoint. Ex post asymmetric information does not undermine efficiency under ex ante contracting thanks to the fact that each interest group can make the decision-maker residual claimant for the consequences of the policy on their bilateral payoff. Our efficiency result is reminiscent of the literature on common agency under complete information but is slightly more subtle. Under complete information, contributions can be tailored to the realized state of nature $\theta$ and without loss of generality can be restricted to be non-negative. This is no longer the case under ex ante contracting. The fixed-fees of each principal's contribution cannot be conditioned on $\theta$, making it more 
difficult to ensure that the grand-coalition of principals always forms and thus that the efficient policy ends up being chosen by the agent. ${ }^{12}$ Nevertheless, the constants $C_{1}$ and $C_{2}$ can be chosen so that none of the principals wants to deviate away from a common representation and induce thereby the agent to serve him exclusively. As a result, the grand-coalition still forms.

Turning now to the distribution of the political surplus, the second important result of our analysis is that the nature of the conflict between interest groups changes with the extent of ideological uncertainty, the degree of polarization and the degree of asymmetrical between groups. As uncertainty shrinks or principals are more polarized, the agent's ideal point is better known to be located around zero and certainly always far away from the principals' own ideal points since principals are significantly biased on both sides of the policy space. Principals compete head-to-head for the agent's services. ${ }^{13}$

When ideological uncertainty is more pronounced or when the degree of polarization is small enough, principals jointly succeed in extracting the agent's ex ante rent. Lobbying competition is somewhat weakened since now principals become more congruent. There is now always a positive probability that both principals' ideal points lie on the same side of the agent's ideal point. On average, principals look more alike. The existing difference between the principals' ideal points is now offset by their common willingness to limit the agent's ex ante rent. However, doing so entails now a coordination problem. Many possible ways of sharing the political surplus are available as long as the agent's deviations towards serving exclusively one of those principals are prevented.

For a fixed level of uncertainty, the more pronounced is the bias parameter towards one principal, the more likely it is that principals are conflicting $\left(\delta^{*}(a, h, \beta)\right.$ increases with $h$ ). As it can be seen from the expressions for the payoffs $C_{i}$, the principal $P_{1}$ who is ideologically far away from the decision-maker suffers more from the asymmetry.

Let us now suppose that entry in the lobbying process is endogenous, at least for $P_{2}$ who may face some extra cost $k$ of entering the political arena. When inactive, this interest group suffers from the policy of the complementary coalition being implemented. There is a threshold $k^{*}$ beyond which entry won't occur. This cut-off decreases with the extent of ideological uncertainty or with the ideological bias of the decision-maker, and it

\footnotetext{
${ }^{12}$ See the Appendix.

${ }^{13}$ To build intuition, we might think about the extreme case where the agent's ideal policy is known for sure $(\delta=0)$. The two principals being symmetrically located around the agent's ideal policy, none of them can really succeed in inducing the agent to choose a policy close enough to his own ideal point even though each principal is ready to significantly bid for doing so. As ideological uncertainty slightly increases, the same head-to-head competition still prevails. Under ex ante contracting, lobbying competition has no impact on the policy chosen which remains efficient as a result of the agent being offered truthful contributions. The only impact of head-to-head competition is that the agent gets most of the surplus of the political process by threatening each principal to deal only with the other. As a result, the agent secures a positive ex ante rent at equilibrium.
} 
increases with the asymmetry bias.

This shows that the pluralistic view of politics is warranted only in environments where ideological uncertainty is more pronounced and groups are close to be symmetrically located at an equal distance of the decision-maker.

\section{$5 \quad$ Appendix}

- Proof of Lemma 1: The proof is standard and thus omitted.

- Proof of Proposition 1: Note that $t_{i}(q)=-\frac{1}{2}\left(q-a_{i}\right)^{2}-C_{i}$ is a contribution which is accepted by the common agent as long as $C_{i}$ is small enough, so that the participation constraints (3) is satisfied. The corresponding condition is explicit below.

For the time being note that such truthful schedule satisfies also the incentive constraints in Lemma 1 and is thus a best-response to the truthful schedule offered by $P_{-i}$. Given that both principals offer truthful schedules, the agent chooses an efficient policy

$$
q^{e a}(\theta)=q^{F B}(\theta)=\arg \max _{q \in \mathbb{R}}\left\{-\sum_{i=1}^{2} \frac{1}{2}\left(q-a_{i}\right)^{2}-\frac{\beta}{2}(q-\theta)^{2}-\sum_{i=1}^{2} C_{i}\right\}=\frac{\beta \theta+h}{\beta+2} .
$$

At a best-response, principal $P_{i}$ increases $C_{i}$ up to the point where (3) binds. This yields the following condition:

$$
W_{12}-\sum_{i=1}^{2} C_{i}=\max \left\{0, W_{i}-C_{i}\right\}, \quad \text { for } i=1,2 .
$$

For further references, note that

$$
W_{12}(\theta)=-a(a+h)-\frac{(\beta+1) h^{2}}{2(\beta+2)}-\frac{\beta \theta(\theta-h)}{\beta+2} \text {, and } W_{i}(\theta)=-\frac{\beta}{2(\beta+1)}\left(a_{i}-\theta\right)^{2} .
$$

Taking expectations, we have

$$
W_{12}=-a(a+h)-\frac{(\beta+1) h^{2}}{2(\beta+2)}-\frac{\beta \delta^{2}}{3(\beta+2)}, \text { and } W_{i}=-\frac{\beta\left(3 a_{i}^{2}+\delta^{2}\right)}{6(\beta+1)} .
$$

- The function $W_{S}$ is superadditive if and only if $W_{12}>\sum_{i=1}^{2} W_{i}$ or $\delta>\sqrt{\frac{3(\beta+2)}{\beta}(a+h) a+\frac{3 h^{2}}{2 \beta}}$.

- When uncertainty on the agent's ideal point is large enough, there exists a continuum of truthful equilibria with payoffs for the principals $\left(C_{1}, C_{2}\right)$ satisfying $(\mathrm{A} 1)$, or to put it differently:

$$
C_{1}+C_{2}=W_{12} \text {, and } W_{i} \leq C_{i} \leq W_{12}-W_{-i}
$$


Note that, for any of those equilibria, the agent's ex ante rent is zero.

- When $\delta \leq \sqrt{\frac{3(\beta+2)}{\beta}(a+h) a+\frac{3 h^{2}}{2 \beta}}$, i.e., when uncertainty on the agent's ideal point is small enough, we have $W_{12} \leq W_{1}+W_{2}$ and $W_{S}$ is subadditive. The unique solution to (A1) is then

$$
C_{i}=W_{12}-W_{-i}
$$

- Let us show now that the agent always choose to take both contracts, i.e., given that $P_{-i}$ offers himself a truthful schedule such that $W_{12}-W_{i} \geq C_{-i}, P_{i}$ cannot profitably deviate by inducing the agent to serve him exclusively.

The first observation is that, under ex ante contracting, the payoff for $P_{i}$ of inducing an exclusive deviation from the agent such that the agent serves this principal only is weakly dominated by the solution to the following problem:

$$
\begin{gathered}
\left(\mathcal{P}_{i}^{\text {ed }}\right): \max _{\left\{q_{i}(\cdot), U_{i}(\cdot)\right\}} \int_{-\delta}^{\delta}\left\{-\frac{1}{2}\left(q_{i}(\theta)-a_{i}\right)^{2}-\frac{\beta}{2}\left(q_{i}(\theta)-\theta\right)^{2}-U_{i}(\theta)\right\} \frac{d \theta}{2 \delta} \\
\quad \text { subject to }(A 5) \\
\dot{U}_{i}(\theta)=\beta\left(q_{i}(\theta)-\theta\right), \quad \dot{q}_{i}(\theta) \geq 0 .
\end{gathered}
$$

and

$$
\int_{-\delta}^{\delta} U_{i}(\theta) \frac{d \theta}{2 \delta} \geq \max \left\{0, \int_{-\delta}^{\delta} U_{-i}(\theta) \frac{d \theta}{2 \delta}\right\}
$$

Indeed, $P_{i}$ 's payoff $C_{i}^{e d}$ of inducing an exclusive deviation can only be lower that the maximal payoff $\tilde{C}_{i}$ achieved at a solution to $\left(\mathcal{P}_{i}^{e d}\right)$ since we have neglected a constraint saying that the agent should prefer to take only $P_{i}$ 's offer than both contracts.

Note then that the incentive compatibility constraints (A5) are satisfied at no cost when $P_{i}$ offers also a truthful schedule $t_{i}(q)=-\frac{1}{2}\left(q-a_{i}\right)^{2}-\tilde{C}_{i}$ since it aligns the agent's objectives with those of the bilateral coalition he forms with this principal. Given that $P_{-i}$ offers also a truthful contribution $t_{-i}^{*}(q)=-\frac{1}{2}\left(q-a_{-i}\right)^{2}-C_{-i}$, the maximal payoff $\tilde{C}_{i}$ is achieved when (A6) is binding, i.e., $W_{i}-\tilde{C}_{i}=\max \left\{0, W_{-i}-C_{-i}\right\}$. Two cases should now be considered.

Congruent principals: Then, $W_{1}+W_{2} \leq W_{12}$. We know that $C_{-i} \geq W_{12}-W_{i}$ and thus $\tilde{C}_{i} \leq W_{i}$ but from (A3), $\tilde{C}_{i}$ is dominated by the payoff with a common representation.

Conflicting principals: Then, $W_{1}+W_{2}>W_{12}$. We know that $C_{-i}=W_{12}-W_{i}$ and thus $\tilde{C}_{i} \leq W_{12}-W_{-i}$ but from (A4), $\tilde{C}_{i}$ is dominated by the payoff with a common representation.

In both cases, deviations to an exclusive representation are always dominated. 


\section{References}

Austen-Smith, D. and J. Banks, 2000. Positive Political Theory 1: Collective Preferences. (Michigan University Press).

Bernheim, D. and M. Whinston, 1986. Menu Auctions, Resource Allocations and Economic Influence, Quarterly Journal of Economics 101, 1-31.

Dahl, R., 1961. Who Governs? Democracy and Power in an American City. (Yale University Press, New Haven)

Grossman, G. and E. Helpman, 1994. Protection for Sale, American Economic Review 84, 833-850.

Horn, M., 1995. The Political Economy of Public Administration: Institutional Choice in the Public Sector. (Cambridge University Press).

Laffont, J.J. and D. Martimort, 2002. The Theory of Incentives: The Principal-Agent Model. (Princeton University Press, Princeton).

Laussel, D. and M. Le Breton, 1998. Efficient Private Production of Public Goods under Common Agency, Games and Economic Behavior 25, 194-218.

Laussel, D. and M. Le Breton, 2001. Conflict and Cooperation: The Structure of Equilibrium Payoffs in Common Agency, Journal of Economic Theory 100, 93-128.

Martimort, D. and A. Semenov, 2006. Political Biases in Lobbying under Asymmetric Information, Journal of European Economic Association, forthcoming.

Mitra, D., 1999. Endogenous Lobby Formation and Endogenous Protection, American Economic Review 89, 1116-1134. 